\title{
INSTITUCIONES, AGENTES Y REFORMAS ESTRUCTURALES. LAS REFORMAS ENERGÉTICAS DE 2008 Y 2013 EN PERSPECTIVA COMPARADA
}

\author{
Germán Petersen Cortés ${ }^{1}$
}

¿QUÉ EXPLICA EL CARÁCTER INCREMENTAL o radical de una reforma estructural: las instituciones o los agentes? El presente trabajo intenta responder esta pregunta. Se trata de una interrogante teórica, la cual, atendiendo a su propia naturaleza, tiene que ser respondida de manera abstracta. Para aportar elementos empíricos que permitan responderla con evidencia se comparan dos casos: los procesos de decisión de las reformas energéticas de 2008 y 2013. Las instituciones que aquí se analizan son formales, es decir, cimentadas jurídicamente. Se entiende la decisión como aquellos procesos en los que se involucraron Ejecutivo y Legislativo para lograr la aprobación de la reforma. Así, cuando se habla de las instituciones directamente implicadas en el proceso de decisión de la reforma, se está refiriendo, específicamente, al Ejecutivo, al Legislativo y a su relación. ${ }^{2}$

Como se verá, esta comparación provee muchos más elementos a favor de los agentes como explicación del carácter incremental

${ }^{1}$ El autor agradece los comentarios del Dr. José Luis Méndez Martínez y de dos dictaminadores anónimos. La investigación se hizo durante los estudios de Maestría del autor, a lo largo de los cuales fue becario de Conacyt, institución a la cual hace patente su agradecimiento.

${ }^{2}$ Otros aspectos de la reforma energética, como las estrategias de comunicación gubernamental para promoverla, las movilizaciones sociales en su contra y la recepción que tuvo en la opinión pública exceden los límites de este trabajo. 
o radical de una reforma, que a favor de las instituciones. La pregunta que guía la investigación empírica, y que es una operacionalización de la pregunta teórica, es: ¿qué explica que se haya aprobado una reforma en materia energética incremental en 2008 -de ajustes en el margen- y una radical en 2013 -de transformaciones de fondo-?

Dentro de la discusión pública mexicana se ha dado por llamar "reformas estructurales" a aquellas modificaciones a la Constitución o a leyes secundarias en aspectos clave para el futuro país, sobre todo en términos económicos. Por lo general, el término se utiliza sin precisar si lo estructural de los cambios se relaciona con la materia de la cual tratan o con la profundidad de las modificaciones. La reforma energética suele entenderse como parte de las reformas estructurales, aunque podría debatirse si también es válido calificar de esta manera una reforma como la de 2008, que, a diferencia de la de 2013, no implicó cambios constitucionales. En el presente trabajo se asume que la reforma de 2008 es estructural, pues, aunque no supuso cambios en la Constitución ni fue radical, se enfocó en un sector fundamental para la economía del país.

La comparación entre ambas reformas se hace desde un diseño de casos "más similares", basado "en la creencia de que los sistemas lo más parecidos posible con respecto a tantas características como sea posible constituyen las muestras óptimas para la investigación comparada". ${ }^{3}$ Conviene el diseño "más similares" porque son muchas más las semejanzas entre las dos unidades de análisis que sus diferencias. Se trata del mismo país -lo que de suyo implica un enorme parecido entre ambas unidades-, hay apenas cinco años de diferencia entre las dos reformas y, además, como se verá, no hubo grandes modificaciones en las instituciones que norman la relación Ejecutivo-Legislativo entre 2008 y 2013 y tampoco en la representación legislativa del partido del presidente. Así, dado que las unidades de análisis son similares y los resultados diferentes -reforma incremental en 2008 y radical en 2013-, la explicación del carácter distinto de ambas reformas se encuentra en las variables que adoptaron

${ }^{3}$ A. Przeworski y H. Teune, The Logic of Comparative Social Inquiry, Nueva York, Wiley, 1970, p. 32. 
un valor diferente en un proceso y en otro. Se parte de la hipótesis de que el comportamiento de los agentes, más que las instituciones, en particular aquellas que norman la relación Ejecutivo-Legislativo, explica que se haya decidido una reforma incremental en 2008 y una radical en 2013. La variable dependiente es el carácter de las reformas -incremental en 2008 y radical en 2013- y las independientes son las instituciones y el comportamiento de los agentes.

Hay que advertir una dificultad de comparabilidad: mientras que la reforma de 2008 es una reforma terminada, cuyas modificaciones no sólo son conocidas por todos sino que ya se pusieron en práctica, la de 2013 es una reforma en proceso, pues aunque ya se aprobó la reforma constitucional, las leyes secundarias y reformas a otras leyes secundarias, éstas aún se están implementando. Para efectos de esta investigación, dicho problema no es grave, pues interesa el carácter incremental de la reforma de 2008 y el radical de la de 2013, mucho más que su contenido específico. El carácter incremental de la primera de estas reformas y el radical de la segunda son ya un hecho, independientemente de la reglamentación de la de 2013.

El texto está compuesto por seis secciones. La primera es de discusión teórica: ¿son las instituciones o el comportamiento de los agentes lo que explica el cambio en la política pública? En el siguiente apartado se argumenta sobre qué base se considera incremental la reforma de 2008 y radical la de 2013. Las dos secciones que siguen son de análisis empírico, la primera sobre la decisión de la reforma de 2008 y la segunda sobre la de 2013. Posteriormente, se comparan ambos procesos de decisión desde un diseño de investigación "más similares". A partir de los hallazgos de esta comparación, se responde, en las conclusiones, la pregunta teórica planteada al inicio.

\section{INSTITUCIONES O AGENTES: EXPLICACIONES DEL CAMBIO EN LA POLÍTICA PÚBLICA}

Desde el punto de vista del campo de políticas públicas, el debate sobre el carácter incremental o radical de una reforma jurídica es parte de la discusión más general en torno al 
cambio. ${ }^{4}$ ¿Qué explica el carácter incremental o radical del cambio en una política pública? Conviene aquí una aclaración: al explicar el cambio, se explica también -aunque sea tácitamente y sólo $a$ contrario- la continuidad. En términos generales, hay dos grandes explicaciones del contenido de una política pública así como de sus cambios: las instituciones y los agentes. Este debate es la expresión en el campo de políticas públicas de la añeja polémica en la teoría social sobre qué lleva la prioridad causal de los fenómenos sociales: la estructura, la institución o el sistema-según la tradición sociológica de que se trate-, o bien la agencia, las prácticas o la acción. La discusión está estrechamente relacionada con la polémica entre el funcionalismo y el estructuralismo por un lado -que se enfocan más en las instituciones y las estructuras-, y la hermenéutica y la sociología comprehensiva por el otro -que se centran más en el agente, su entendimiento del mundo y sus acciones-. ${ }^{5}$

Dentro de quienes colocan el acento en las instituciones se encuentran March y Olsen, ${ }^{6}$ Mahoney y Thelen, ${ }^{7}$ y Heclo. ${ }^{8}$ En oposición a ellos están quienes se enfocan en los agentes, pero sin soslayar la importancia de las instituciones. Ahí se pueden ubicar autores como Schattschneider, ${ }^{9}$ Bachrach y Baratz, ${ }^{10}$ y Hassenteufel, Smyrl, Genieys, y Moreno-Fuentes. ${ }^{11}$

${ }^{4}$ Desde otras disciplinas, como el derecho constitucional, u otras áreas de investigación, como los estudios legislativos, la explicación del cambio es diferente. A lo largo de este trabajo se adopta siempre el punto de vista del campo de políticas públicas.

${ }^{5}$ Una estupenda y breve síntesis de este problema aparece en A. Giddens, $L a$ constitución de la sociedad, Buenos Aires, Amorrortu, 2011, pp. 39-40.

${ }^{6} \mathrm{~J}$. March y J. Olsen, Rediscovering Institutions: The Organizational Basis of Politics, Nueva York, The Free Press, 1989.

${ }^{7}$ J. Mahoney y K. Thelen, "A Theory of Gradual Institutional Change”, en J. Mahoney y K. Thelen (eds.), Explaining Institutional Change, Nueva York, Cambridge University Press, 2010, pp. 1-37.

${ }^{8} \mathrm{H}$. Heclo, "Las redes de asuntos y el poder Ejecutivo", en L. Aguilar (ed.), Problemas públicos y agenda de gobierno, México, Miguel Ángel Porrúa, 2000, pp. 257-284.

${ }^{9}$ E. Schattschneider, El pueblo semisoberano, México, Unión Tipográfica Hispanoamericana, 1967.

${ }^{10}$ P. Bachrach y M. Baratz, "Two Faces of Power", The American Political Science Review, vol. 56, núm. 4, 1962, pp. 947-952.

11 P. Hassenteufel, M., Smyrl, W. Genieys y F. Moreno-Fuentes, "Program- 
March y Olsen ${ }^{12}$ y Mahoney y Thelen ${ }^{13}$ estudian las instituciones, no directamente las políticas públicas, mientras Heclo ${ }^{14}$ sí se concentra específicamente en estas. Desde las categorías de Hall y Taylor, ${ }^{15}$ si bien tanto March y Olsen como Mahoney y Thelen son neoinstitucionalistas, el primero es sociológico y el segundo histórico. March y Olsen sostienen que el "comportamiento está gobernado por reglas" y, en términos políticos, estas reglas son "rutinas, procedimientos, convenciones, roles, estrategias, formas organizacionales y tecnologías alrededor de las cuales se construye la actividad política" ${ }^{16}$ Así, las instituciones no sólo son el marco en el que se desarrolla la política, sino que también modelan las preferencias de los agentes. Tal modelación de preferencias se hace mediante lo que los autores llaman "lógica de lo apropiado", es decir, "lo que es apropiado para una persona particular en una situación particular", y que es "transmitido mediante la socialización". ${ }^{17} \mathrm{El}$ corolario de esto para las políticas públicas es que hay un número limitado de opciones de política, definido por las instituciones.

Mahoney y Thelen tratan de explicar el cambio institucional, después de detectar las deficiencias del neoinstitucionalismo para hacerlo. Según su planteamiento, hay escenarios institucionales que inducen a los agentes hacia cierto comportamiento. A diferencia de otros planteamientos, que conciben a las instituciones como continuas reforzadoras de sí mismas, ${ }^{18}$ estos autores destacan que las instituciones pueden propiciar contextos que induzcan al cambio. De esta manera, Mahoney y Thelen sostienen que el cambio

matic Actors and the Transformation of European Health Care States", Journal of Health Politics, Policy and Law, vol. 35, núm. 4, 2010, pp. 517-538.

12 March y Olsen, op. cit.

${ }_{13}$ Mahoney y Thelen, op. cit.

${ }^{14}$ Heclo, op. cit.

${ }^{15}$ P. Hall, y R. Taylor, "Political science and the three new institutionalisms", Political Studies, núm. 44, 1996, pp. 936-957.

${ }^{16}$ March y Olsen, op. cit., p. 22.

17 Ibid., p. 23.

18 D. North, Instituciones, cambio institucional y desempeño económico, México, FCE, 1993; D. North, "Toward a Theory of Institutional Change”, en W. Barnett, N. Schofield y M. Hinich, (eds.), Political Economy: Institutions, Competition, and Representation, Cambridge, Cambridge University Press, 1993, pp. 61-69. 
institucional no necesariamente es provocado por una variable exógena ni se da siempre de manera repentina, sino que puede ser endógeno y progresivo, propulsado por alteraciones en la coalición que sostiene a la institución, en la relación entre instituciones y en el grado de conformidad (compliance) de los agentes.

Heclo ${ }^{19}$ advierte que las concepciones políticas estándar, basadas en "triángulos de hierro" (burocracia, comités del Congreso y grupos de interés), "no son adecuadas para entender este nuevo juego fragmentado de influencias que tiene lugar en la administración de las políticas". ${ }^{20}$ Como alternativa propone estudiar las políticas públicas a partir de redes de asuntos, en las que se insertan los "triángulos de hierro" y muchos otros grupos. En estas redes, "los participantes entran y salen constantemente [...] más que [ser] grupos unidos para tener dominio sobre un programa, no hay nadie que de hecho controle las políticas y los problemas". ${ }^{21}$ En último término, para Heclo el tipo de red define el tipo de política pública, no los triángulos de hierro ni determinados agentes en particular.

Los enfoques que colocan el énfasis en las instituciones para explicar el cambio en las políticas públicas resultan ventajosos para estudiar la continuidad, pero no el cambio. Según March y Olsen, las instituciones, además de regular el comportamiento de los agentes, modelan sus preferencias, lo cual limita enormemente la posibilidad de esta teoría para explicar el cambio, cuando no sea por un shock externo. Heclo se concentra en la importancia de las redes para la configuración de las políticas, sugiriendo que el cambio en el contenido de una política pública sólo puede explicarse como consecuencia del cambio en el tipo de red. Mahoney y Thelen son quienes, entre estos tres textos, explican mejor el cambio institucional. Sin embargo, al final del día, subrayan cómo las

${ }^{19}$ Heclo, op. cit.

${ }^{20} \mathrm{Ibid}$., p. 262. Se cita la traducción que aparece en la antología editada por Luis F. Aguilar, a cargo de Graciela Bellón Pérez, que es la más usual, aunque cabe aclarar que la edición en inglés alude a un juego loose-jointed, cuya traducción más adecuada sería "suelto-articulado", no "fragmentado", como lo traduce Bellón.

${ }^{21}$ Heclo, op. cit., p. 263. 
instituciones generan contextos propicios para el cambio, pero terminan asociando el cambio en sí, además de con las instituciones, con el comportamiento de los agentes -cambios en la coalición dominante o variaciones en el grado de conformidad de los agentes con la institución- o con factores exógenos.

En oposición a la perspectiva institucionalista, está la enfocada en los agentes. Schattschneider, exponente de esta segunda perspectiva, sostiene que "en el meollo de la política se encuentra [...] la manera en que participa el público en la propagación del conflicto, y $[\ldots]$ el proceso por medio del cual se controlan las relaciones inestables del público y el conflicto". ${ }^{22}$ A su parecer, "el resultado de las contiendas políticas está determinado por el alcance de la participación pública en los conflictos". ${ }^{23}$ De aquí se desprende el carácter estratégico de la política: si el alcance de la participación pública en los conflictos determina el resultado, los agentes interesados en ganar deben comportarse estratégicamente en la propagación o limitación del conflicto. Más aún, la estrategia de los agentes no sólo es clave para lograr un objetivo posible, sino también para ampliar el alcance de lo posible y, después, aspirar a un objetivo que inicialmente parecía imposible.

Bachrach y Baratz, ${ }^{24}$ desde una posición muy cercana a la de Schattschneider, lanzan fuertes críticas a los conceptos de poder de su tiempo, específicamente al clásico de Dahl, ${ }^{25}$ pues sostienen que estos sólo consideran una cara del poder -la más aparente, relacionada con quién ejerce el poder- pero relegan otra, la menos aparente, asociada con la manera como se define un asunto determinado. La definición de cualquier asunto limita las opciones de acción disponibles y, por ende, condiciona la toma de decisiones futuras. En términos metodológicos, Bachrach y Baratz proponen cuatro pasos para el estudio de las dos caras del poder: a) investigar cómo se está "movilizando el sesgo" (expresión que

${ }^{22}$ Schattschneider, op. cit., p. 4.

23 Ibid., p. 7.

${ }^{24}$ Bachrach y Baratz, op. cit.

25 "A tiene poder sobre B en la medida en que pueda conseguir que B haga algo que de otra manera no haría" (R. Dahl, "The Concept of Power", Behavioral Science, vol. 2, núm. 3, 1957, pp. 202-203). 
retoman de Schattschneider) en la institución bajo estudio, es decir, cómo se está definiendo el asunto en cuestión; $b$ ) dilucidar cuáles personas o grupos se benefician del sesgo y cuáles son afectados por éste; $c$ ) investigar la dinámica de la no toma de decisión, es decir, investigar el grado y la manera en que el statu quo limita el espectro de opciones; y, finalmente, $d$ ) analizar la toma de decisiones en un asunto en concreto. ${ }^{26}$

Hassenteufel, Smyrl, Genieys y Moreno-Fuentes ${ }^{27}$ estudian los actores programáticos, es decir, "actores colectivos que comparten ideas de políticas y compiten por la autoridad legítima sobre la formulación de políticas sectoriales". ${ }^{28}$ "Estos 'actores programáticos' son importantes impulsores del cambio de política y, en particular, son los principales determinantes del contenido de la política". ${ }^{29}$ A diferencia del concepto de coaliciones promotoras, propuesto por Sabatier y Jenkins-Smith, ${ }^{30}$ aplicable a aquellos grupos que buscan dominar un subsistema de políticas, los actores programáticos tienen como objetivo la formulación de una política sectorial en concreto, no el dominio prolongado sobre un subsistema. La composición de los actores programáticos puede ser muy diversa, siempre y cuando coincidan en una misma idea de políticas y estén dispuestos a impulsarla.

Los enfoques que se centran en los agentes para explicar el cambio en las políticas públicas son mucho más útiles que los centrados en las instituciones. Dado que los enfoques que priorizan a los agentes asumen que estos tienen libertad para actuar, la transformación del statu quo se explica como consecuencia de esta libertad. Los autores citados coinciden en que la estrategia de los agentes es determinante para que estos logren sus objetivos mediante el ejercicio de poder, entendido éste no sólo como que A logre que B haga lo que A quiere -a la manera de Dahl-, sino también como la definición de un asunto determinado -"movilización

${ }^{26}$ Bachrach y Baratz, op. cit., p. 952.

${ }^{27}$ Hassenteufel, Smyrl, Genieys, y Moreno-Fuentes, op. cit.

28 Ibid., p. 518.

${ }^{29}$ Ibid., p. 519.

${ }^{30}$ P. Sabatier, y H. Jenkins-Smith, "The Advocacy Coalition Framework", en P. Sabatier (ed.), Theories of the Policy Process, Boulder, Westview Press, 1999, pp. 117-166. 
del sesgo", siguiendo a Schattschneider-, de manera tal que A saque ventaja de las posibilidades de acción que abrió la definición que impuso y también de las que cerró. Aun cuando los autores correspondientes a este enfoque concentran mayor atención en los agentes, no soslayan la importancia de las instituciones. Las instituciones son el marco en el que sucede la propagación del conflicto para Schattschneider, la movilización del sesgo para el propio Schattschneider y para Bachrach y Baratz, y donde operan los actores programáticos de Hassenteufel, Smyrl, Genieys y Moreno-Fuentes.

\section{DE LA REFORMA INCREMENTAL A LA REFORMA RADICAL}

El cambio incremental es el que surge de lo que Lindblom denomina método de comparaciones limitadas sucesivas o método de irse por las ramas, que se opone al método racional-exhaustivo o método de ir a la raíz. ${ }^{31} \mathrm{El}$ método de irse por las ramas, "al decidir a través de una sucesión de cambios incrementales, evita de diversas maneras errores serios". ${ }^{32}$ Este método pretende, como el mismo Lindblom señala, "salir del paso", "nada más que salir del aprieto". 33

Quade, ${ }^{34}$ por su parte, defiende el método racional en el diseño de políticas, partiendo de que estas deben servir para lograr cambios deseables en el estado de las cosas. Por ello, relaciona el análisis de políticas con la investigación de operaciones y el análisis de sistemas, aunque con el añadido de que las políticas, a diferencia de las operaciones y los sistemas, deben buscar la equidad. "La investigación de operaciones busca (o buscaba) contribuir a realizar un objetivo dado de la mejor manera; el análisis de sistemas busca lo mismo, pero trata de realizar la cosa correcta y de la manera más económica; el análisis de políticas busca realizar lo

${ }^{31}$ C. Lindblom, "La ciencia de 'salir del paso'”, en L. Aguilar (ed.), La hechura de las políticas, México, Miguel Ángel Porrúa, 2000, pp. 201-225.

32 Ibid., p. 220.

33 C. Lindblom, "Todavía tratando de salir del paso", en L. Aguilar (ed.), La hechura de las políticas, México, Miguel Ángel Porrúa, 2000, p. 227.

${ }^{34}$ E. Quade, Analysis for Public Decisions, Nueva York, American Elsevier, 1975. 
mismo que el análisis de sistemas, pero con equidad". ${ }^{35}$ A diferencia de Lindblom, Quade deja abierto el espacio para que se formulen políticas radicales, no sólo incrementales, si se juzga que lo deseable es una transformación de fondo.

El 28 de noviembre de 2008, el entonces presidente Felipe Calderón promulgó los siete decretos que integraron la reforma energética de aquel año. Cuatro de estos decretos expedían igual número de nuevas leyes -Ley de Petróleos Mexicanos (pemex), Ley de la Comisión Nacional de Hidrocarburos (CNH), Ley para el Aprovechamiento de Energías Renovables y el Financiamiento de la Transición Energética y Ley para el Aprovechamiento Sustentable de la Energía- y tres más reformaban, adicionaban o derogaban leyes existentes -Ley Reglamentaria del Artículo 27 Constitucional en el Ramo del Petróleo, Ley de la Comisión Reguladora de Energía (CRE) y Ley Orgánica de la Administración Pública Federal-. ${ }^{36}$

La nueva Ley de PEMEx disponía de cambios corporativos en la empresa, los más relevantes de los cuales fueron la inclusión de cuatro consejeros profesionales en el Consejo de Administración y una nueva estructura para este órgano. Además, dotaba a la empresa de mayor autonomía, especialmente en el manejo de bonos, deuda, presupuesto, contrataciones y otras cuestiones administrativas, aunque también la obligaba a mayor rendición de cuentas frente al Congreso y la SHCP. La Ley de la CNH creaba esta Comisión y la instauraba como el órgano regulatorio y supervisor de la exploración y extracción de hidrocarburos, buscando que ambos procesos fueran eficientes, convenientes a los intereses financieros del Estado, sostenibles en el tiempo, sustentables ecológicamente y seguros industrialmente.

La Ley para el Aprovechamiento de Energías Renovables y el Financiamiento de la Transición Energética se colocaba como objetivo regular la generación tanto pública como privada de electricidad, buscando que fuera a partir de energías renovables y tecnologías

${ }^{35}$ Quade, op. cit., p. 22.

36 Diario Oficial de la Federación, 28 de noviembre de 2008. Consultado el 7 de septiembre de 2015, en http://dof.gob.mx/nota_to_doc.php?codnota=5070925 
limpias. En breve, establecía las coordenadas para que el país transitara hacia un modelo de energía eléctrica de menor impacto ambiental. De manera semejante, la Ley para el Aprovechamiento Sustentable de la Energía promovía el uso óptimo de los energéticos, con especial atención a medidas a favor de la eficiencia, como certificados.

En cuanto a las leyes reformadas, los cambios a la Ley Reglamentaria del Artículo 27 Constitucional en el Ramo del Petróleo adecuaban el marco normativo de la industria petrolera a las nuevas condiciones globales, estableciendo con mayor claridad los alcances de la legislación mexicana y de la internacional. Mediante la reforma a la Ley de la cRE se incrementó el número de actividades reguladas por este órgano y se modificó su funcionamiento interno y su relación con el Legislativo. Finalmente, las modificaciones a la Ley Orgánica de la Administración Pública Federal contemplaron nuevas facultades para la Secretaría de Energía, apuntalándola como la conductora de la política energética del país, no como una jugadora en el mercado de energía.

El 20 de diciembre de 2013, el presidente Enrique Peña Nieto promulgó el decreto mediante el cual se reformaron tres artículos de la Constitución -25, 27 y 28- en sus contenidos relativos a energía y se expedían 21 artículos transitorios. ${ }^{37}$ La reforma al artículo 25 introdujo el término "empresas productivas del Estado" para PEMEX y la Comisión Federal de Electricidad (CFE). A partir de entonces, ambas empresas se tendrían que atener a principios de eficacia, eficiencia, honestidad, productividad, transparencia y rendición de cuentas. Además, se introdujo el criterio de sustentabilidad como clave en las industrias eléctrica y de hidrocarburos.

Las modificaciones al artículo 27 acabaron con el monopolio público en electricidad, con que se dejó en manos del Estado, de manera exclusiva, la planeación y el control de la industria eléctrica. Si bien se mantuvo la histórica prohibición a las concesiones en transmisión y distribución de electricidad, se abrió la posibilidad a

37 Diario Oficial de la Federación, 20 de diciembre de 2013. Consultado el 7 de septiembre de 2015, en http://www.dof.gob.mx/nota_detalle.php?codigo=53274 $63 \&$ fecha $=20 / 12 / 2013$ 
la celebración de contratos entre el Estado y particulares en las demás actividades de la industria eléctrica. De manera semejante, se mantuvo la negativa a concesiones en materia de hidrocarburos, pero se permitió que tanto la exploración como la explotación se asignaran a empresas productivas del Estado o se celebraran contratos con estas o con particulares.

Por último, la reforma el artículo 28 creó el Fondo Mexicano del Petróleo para la Estabilidad y el Desarrollo, encargado de administrar los recursos derivados de las asignaciones y contratos establecidos en el artículo 27 y que desde su creación tiene como fiduciario al Banco de México. Asimismo, se subieron a rango constitucional la CNH y la CRE.

En los artículos transitorios se estableció un plazo de dos años para que PEMEX y la CFE se conviertan en empresas productivas del Estado. Además, y de manera muy relevante, se crearon cuatro distintas las modalidades de contratación entre el Estado y los particulares en exploración y extracción de hidrocarburos: de servicios, de utilidad compartida, de producción compartida y de licencia. A partir de entonces, las empresas en el mercado energético, tanto públicas como privadas, pueden reportar, para efectos contables, las asignaciones o contratos con los que contaran. Quedó reafirmada la posición de la Secretaría de Energía como la conductora de la política energética del país y por ende como la institución encargada de hacer las asignaciones y celebrar los contratos. Los transitorios también otorgaron al sector energético el carácter de interés social, conque lo ubicaron como prioritario en el uso del suelo y el subsuelo.

Unos meses después de promulgada la reforma constitucional, el 11 de agosto de 2014, se expidieron nuevas leyes ordinarias en materia energética -Ley de la Industria Eléctrica, Ley de Energía Geotérmica, Ley de Órganos Reguladores Coordinados en Materia Energética, Ley de la Agencia Nacional de Seguridad Industrial y de Protección al Medio Ambiente del Sector Hidrocarburos, Ley de Hidrocarburos, Ley de Pemex, Ley de la CRE, Ley del Fondo Mexicano del Petróleo para la Estabilización y el Desarrollo, y Ley de Ingresos sobre Hidrocarburos- y se reformaron, adicionaron y derogaron otras -Ley de Aguas Nacionales, Ley Orgánica de la 
Administración Pública Federal, Ley de Inversión Extranjera, Ley Minera, Ley de Asociaciones Público-Privadas, Ley Federal de las Entidades Paraestatales, Ley de Adquisiciones, Arrendamientos y Servicios del Sector Público, Ley Federal de Derechos, Ley de Coordinación Fiscal, Ley Federal de Presupuesto y Responsabilidad Hacendaria, Ley General de Deuda Pública, y Ley de Obras Públicas y Servicios Relacionados con las Mismas. ${ }^{38}$ Estas nuevas leyes y cambios a normas jurídicas existentes especificaban las reformas constitucionales y los artículos transitorios descritos.

Mientras la reforma de 2008 se limitó a leyes secundarias, la de 2013 modificó tres artículos de la Constitución, además de una cantidad muy significativa de leyes secundarias, lo que da cuenta de la incrementalidad de la primera y la radicalidad de la segunda. $\mathrm{Si}$ bien ambas reformas coinciden en tanto liberalizan actividades económicas antes casi exclusivamente reservadas para el Estado, la reforma de 2008 se limita a crear instituciones regulatorias y técnicas así como a modificar el manejo presupuestario de PEMEx, mientras que la de 2013 abre ampliamente el sector a la inversión privada, impulsa esquemas que dan certidumbre al flujo de capitales, da un giro a PEMEX y a CFE para convertirlas en empresas productivas del Estado, y diseña una nueva arquitectura para regular el sector.

\section{LA REFORMA ENERGÉTICA DE 2008: PROCESO DE DECISIÓN ${ }^{39}$}

La reforma incremental de 2008 fue propuesta por el presidente Felipe Calderón y aprobada, en una versión distinta a la enviada por el Ejecutivo, por amplia mayoría tanto en el Senado como en la Cámara de Diputados. En el Senado, los diferentes cambios que

38 Diario Oficial de la Federación, 11 de agosto de 2014, consultado el 7 de septiembre de 2015, en http:/ / www.dof.gob.mx/nota_to_doc.php?codnota=5355985; Diario Oficial de la Federación, 11 de agosto de 2014. Consultado el 7 de septiembre de 2015, en http://www.dof.gob.mx/nota_to_doc.php?codnota=5355988; Diario Oficial de la Federación, 11 de agosto de 2014. Consultado el 7 de septiembre de 2015, en http://www.dof.gob.mx/nota_to_doc.php?codnota $=5355982$

${ }^{39}$ La información sobre los procesos de decisión de ambas reformas se obtuvo de una revisión de prensa, sobre todo de El Universal. 
comprendió la reforma rondaron los 110 votos a favor -de 128 senadores- y en la Cámara de diputados nunca estuvieron por debajo de los 400 -de 500 diputados-. En términos generales, el apoyo legislativo a la reforma provino del Partido Revolucionario Institucional (PRI), el Partido Acción Nacional (PAN) y en menor medida del Partido de la Revolución Democrática (PRD), más algunos diputados y senadores de partidos pequeños. Quienes votaron en contra fueron algunos legisladores de izquierda, afines a Andrés Manuel López Obrador.

Durante la LX Legislatura (2006-2009), tanto en el Senado como en la Cámara de Diputados hubo gobierno dividido, pues el PAN, partido del presidente, no tenía mayoría absoluta en ninguna cámara, aunque sí contaba con mayoría relativa. En la Cámara Alta, el grupo parlamentario del blanquiazul estaba integrado por 52 senadores, es decir, 40.6\% del total; en la Baja tenía 206 diputados, o sea $41.2 \%$. Que el PAN no contara con la mitad más uno del pleno le obligaba a negociar con otros partidos, incluso para aprobar leyes secundarias, que requieren para su aprobación $50 \%$ más uno de los legisladores presentes. Concretar reformas constitucionales era mucho más complicado, pues precisan de dos terceras partes de los legisladores presentes en ambas cámaras y la mitad más uno de los congresos locales.

En aquella legislatura, el PRI contaba con 33 senadores; el PRD con 26; Partido Verde Ecologista de México (PVEM) con seis; el Partido del Trabajo (PT) con cinco; Partido Convergencia ${ }^{40}$ con cinco; y el Partido Nueva Alianza (PANAL) con uno. Esta composición permitía al PAN y al PRI, votando en bloque, aprobar reformas constitucionales, pues sumaban 85 senadores, es decir, exactamente dos terceras partes del total, cifra suficiente para aprobar una reforma constitucional, toda vez que la propia Carta Magna especifica, en su artículo 135, que basta con dos terceras partes de los presentes, no dos terceras partes más uno. En lo que se refiere a diputados, PAN y PRI, juntos, completaban sólo $62.4 \%$ de los 500

${ }^{40}$ En 2011, el Partido Convergencia (PC) pasaría a llamarse Movimiento Ciudadano (MC), por lo que en los sucesos posteriores a esta fecha se refiere a este como MC. 
diputados -PRI tenía 106; PRD, 127; PT, 11; PC, 18; PVEM, 17; PANAL, 9; y Partido Socialdemócrata (PSD), 5-, por lo que, sólo entre los dos grupos parlamentarios, no podían sacar adelante reformas constitucionales. Para completar las dos terceras partes, lo más viable habría sido incorporar a los partidos tradicionalmente más ligados al PRI, o sea PVEM y PANAL. Estimar la suerte que habría tenido un eventual intento de reforma constitucional en materia energética en los 31 congresos locales y la Asamblea Legislativa del Distrito Federal sería demasiado aventurado, pero baste con decir que, si hubiera contado con el apoyo del PAN y el PRI en el Congreso federal, habría tenido grandes probabilidades de salir adelante en los legislativos locales.

Constitucionalmente, México es un país presidencialista y, en los hechos, el presidente fue preponderante durante prácticamente todo el régimen priista. Como ha argumentado Casar, ${ }^{41} \mathrm{el} \mathrm{am-}$ plio poder presidencial se fundamentaba en la penetración del Ejecutivo en el resto de las instituciones políticas, sobre todo mediante su fuerte control sobre el partido hegemónico. En 1997, el PRI perdió la mayoría absoluta en la Cámara de Diputados y en 2000 en la de Senadores. Se ha debatido ampliamente qué tanto fue el gobierno dividido lo que impidió la aprobación de reformas constitucionales de gran calado durante los sexenios panistas. ${ }^{42}$ Uno de los puntos de acuerdo entre los especialistas es que aprobar reformas constitucionales a partir de 1997 es mucho más complicado, pues la existencia de gobierno dividido obliga al partido del presidente a negociar.

En este contexto, en abril de 2008, Calderón presentó al Senado su iniciativa de reforma energética, que, como se ha dicho, no comprendía cambios constitucionales, sino sólo nuevas leyes secundarias y reformas a algunas ya existentes. Posteriormente, en

${ }^{41}$ M. Casar, "Las bases político-institucionales del poder presidencial en México", Política y Gobierno, vol. 3, núm. 1, 1996, pp. 61-92.

42 M. Casar, "Los gobiernos sin mayoría en México: 1997-2006", Política y Gobierno, vol. 15, núm. 2, 2008, pp. 221-270; M. Casar e I. Marván (coords.), Reformar sin mayorías, México, Taurus, 2014; E. Magar y V. Romero, "México: reformas pese a un gobierno dividido", Revista de Ciencia Política, vol. 28, núm. 1, 2008, pp. 265285; B. Nacif, The Fall of the Dominant Presidency, México, CIDE, 2006. 
mayo, el Senado convocó al foro "Los principios que deben regir la reforma energética en México", al que se dieron cita distintos especialistas. El PAN, al tiempo que defendió la propuesta presidencial, negoció con la oposición la elaboración de una nueva iniciativa. En julio el PRI presentó su iniciativa de reforma, en agosto hicieron lo propio el PRD, el PC y el PT, en una sola iniciativa, y luego el PVEM propuso otra más. A partir de los comentarios recogidos en los foros y de las propuestas de los distintos grupos parlamentarios, el PAN presentó otra iniciativa en septiembre que moderaba las expectativas de la presidencial. Finalmente, "la Comisión de Energía del Senado dictaminó las iniciativas presentadas por todos los grupos parlamentarios representados en el Senado, en los que se incorporaron diversas propuestas expresadas en la consulta pública", ${ }^{43}$ de donde saldría la reforma finalmente aprobada en noviembre de aquel año.

\section{LA REFORMA ENERGÉTICA DE 2013: PROCESO DE DECISIÓN}

La reforma finalmente aprobada en 2013 integró las iniciativas del del Pan y del presidente Enrique Peña Nieto, dejando fuera las de otros partidos, como el PRD. La iniciativa presidencial, apoyada por el PRI, únicamente buscaba reformar los artículos 27 y 28, no el 25. Incluía eliminar la prohibición de que el Estado celebrara contratos con particulares para la explotación, industrialización, transporte, almacenamiento, distribución y comercialización de hidrocarburos, aunque rechazaba el otorgamiento de concesiones. En electricidad, postulaba la apertura de un mercado donde el Estado -salvo por la CFE, que sería una empresa dentro de este mercado- sólo se dedicara a la planeación y el control. ${ }^{44}$

La iniciativa de reforma constitucional del PAN consistía en la modificación de los tres artículos finalmente cambiados. La modificación más polémica se planteaba para el artículo 27: retirar la

43 G. Gil, "La reforma petrolera de 2008 en México. Aspectos jurídicos, efectos económicos", Este País, núm. 214, 2009, p. 40.

${ }^{44}$ El Universal, "Reforma energética propuesta por EPN", 2013. Consultado el 10 de septiembre de 2015, en http://www.redpolitica.mx/contenido/documento-integro-reforma-energetica-propuesta-por-epn 
prohibición a concesiones en hidrocarburos, lo cual fue el punto de mayor diferencia con el PRI y que se resolvió incluyendo una figura intermedia, las licencias. También se buscaba llevar a rango constitucional el marco normativo del Fondo Mexicano del Petróleo, la CNH y la CRE en el artículo 28, y colocar la sustentabilidad como un elemento central del artículo 25.45

La propuesta del PRD, por su parte, no contemplaba la reforma de artículo constitucional alguno, sino sólo modificaciones a leyes secundarias. Entre sus objetivos destacaba resolver los problemas administrativos y financieros de PEMEx, sobre todo dotándola de mayor autonomía presupuestal y de gestión, y reduciéndole la carga fiscal. Proponía también un esquema de acceso más equitativo a la electricidad y los hidrocarburos, mediante modificaciones al programa de subsidios imperante. Se asemejaba a las propuestas priista y panista en tanto contemplaba un Fondo de Estabilización de Ingresos Petroleros. Por último, atendía la necesidad de generar investigación y desarrollo en energía, así como de implementar medidas más efectivas de sustentabilidad, transversales a todas las actividades del sector. ${ }^{46}$

El Senado, primera cámara que aprobó el dictamen que integraba las propuestas del PRI y el PAN, lo hizo con 95 votos a favor y 28 en contra (Senado de la República, 2013); mientras que la Cámara de Diputados lo hizo con 354 a favor y 134 en contra (Cámara de Diputados, 2013). Las votaciones de cada uno de los cambios específicos fueron ligeramente diferentes a la aprobación en lo general. El apoyo a la reforma provino sobre todo de legisladores del PRI, PVEM, PANAL y la inmensa mayoría de los del PAN, y fue rechazada por los partidos de izquierda, es decir, PRD, PT y Movimiento Ciudadano (MC).

45 Grupo parlamentario del PAN en el Senado de la República, "Iniciativa de reforma a los artículos 25, 27 y 28 de la Constitución Política de los Estados Unidos Mexicanos", 2013. Consultado el 10 de septiembre de 2015, en http://www.pan. senado.gob.mx/wp-content/uploads/2013/07/Inic_PAN_art.25-27-y-28-Const.pdf

${ }^{46}$ El Universal, "Propuesta de reforma energética del PRD", 2013. Consultado el 10 de septiembre de 2015, en http://www.redpolitica.mx/contenido/documento-propuesta-de-reforma-energetica-del-prd 
En la LXII Legislatura (2012-2015), actualmente en funciones, el PRI -partido del presidente- no tiene mayoría absoluta en ninguna de las dos cámaras, aunque sí mayoría relativa en ambas. En el Senado, el PRI cuenta con 52 escaños, es decir, $40.6 \%$ del total; el otro $59.4 \%$ está repartido entre PAN (38 escaños), PRD (22), pvem (9), PT (5), mC $\left(1^{47}\right)$ y PANAl (1). En la Cámara de diputados, el PRI cuenta con 212 curules, lo que representa $42.4 \%$ del total; el resto está distribuido entre PAN (114 curules), PRD (104), PVEM (29), MC (16), PT (15) y PANAL (10). Contando a sus partidos tradicionalmente afines -PVEM y PANAL-, el PRI tiene la mayoría absoluta en la Cámara de Diputados, pero no así en la de Senadores. Ante una reforma energética como la de 2013, que de entrada fue rechazada por la izquierda dado que buscaba ampliar la participación de particulares en el sector energético, el PRI sólo podía lograr las dos terceras partes del Congreso sumando al PAN.

Las instituciones que norman las relaciones entre Ejecutivo y Legislativo, en lo que se refiere a presentación, negociación y aprobación de leyes y reformas, en 2013 son casi idénticas a las de 2008. Los principales elementos de la reforma política de 2012 fueron nuevas formalidades a cumplir para la toma de posesión del titular del Ejecutivo y procedimientos a agotar en caso de ausencia del presidente, así como más mecanismos de control de parte del Legislativo sobre las facultades del presidente para nombrar y remover funcionarios de alto nivel. El único cambio incluido en esta reforma que incide sobre el proceso legislativo es la facultad concedida al Ejecutivo para que presente hasta dos iniciativas preferentes en cada periodo ordinario de sesiones, que deberán ser votadas con celeridad por el Congreso o de lo contrario quedarían aprobadas en automático. Salvo por el caso de la iniciativa preferente, la reforma política no tuvo incidencia alguna en los mecanismos de presentación, negociación y aprobación de reformas y mucho menos en la generación de mayorías dentro

${ }^{47}$ A lo largo de todo el proceso bajo estudio, la senadora Layda Sansores fue la única integrante de la fracción de Mc. El 1 de abril de 2014 decidió pasarse al grupo parlamentario del PT, por lo que ahora MC ya no tiene representación en la Cámara alta. 
del Legislativo. Además, la figura de iniciativa preferente ni siquiera fue utilizada por el presidente Peña Nieto al enviar su iniciativa de reforma energética.

El 2 de diciembre de 2012, un día después de que Peña Nieto tomara posesión como presidente, los dirigentes nacionales del PAN y el PRD, Gustavo Madero y Jesús Zambrano, respectivamente, la lideresa nacional del PRI, Cristina Díaz y el presidente de la República firmaron el Pacto por México, un acuerdo entre los principales partidos para impulsar una agenda de reformas estructurales en la que estaban de acuerdo todos los firmantes. A partir del Pacto, se generó un ímpetu reformista sin precedentes desde el salinismo, dentro del cual se aprobaron reformas como la educativa, de telecomunicaciones, de competencia económica y financiera. El acuerdo comenzó a enfrentar inestabilidades a fines de 2013, cuando el PAN se negó a apoyar la reforma fiscal impulsada por el Ejecutivo, que terminó siendo aprobada con su voto en contra. Sin embargo, el punto de quiebre sobrevino cuando, en noviembre, el PRD anunció su salida del Pacto, al declararse en contra de la reforma energética que negociaban los otros dos partidos signatarios del acuerdo.

Si bien en términos estrictos la reforma energética no se aprobó dentro del marco del Pacto por México, sí se benefició ampliamente del espíritu de colaboración detonado por este. El 31 de julio de 2013, los grupos parlamentarios del PAN en el Senado y en la Cámara de Diputados presentaron una ambiciosa propuesta de reforma energética, que apostaba por la transformación de los monopolios públicos en hidrocarburos y electricidad en mercados competitivos. De hecho, esta propuesta inicial del PAN era más radical que la reforma finalmente aprobada, pues contemplaba la figura de concesiones para la explotación de petróleo. El 12 de agosto de 2013, el Ejecutivo federal presentó al Congreso su iniciativa de reforma, que retomaba la formulación del artículo 27 promulgado por Lázaro Cárdenas, y adecuaba el 25 y el 28, orientándolos hacia una mayor participación de los particulares en el sector energético.

De los tres partidos mayoritarios, el último en hacer pública su propuesta de reforma energética fue el PRD, que la presentó el 19 de agosto. No planteaba modificaciones constitucionales, sino 
adecuaciones a leyes secundarias, orientadas a mejorar el funcionamiento del monopolio público en materia de hidrocarburos y electricidad, pero no a desmontarlo. El 29 de noviembre, cuando fue claro que PAN y PRI apostarían por una reforma liberalizadora en materia energética, el PRD abandonó el Pacto por México.

COMPARACIÓN ENTRE LOS PROCESOS DE DECISIÓN DE 2008 y 2013

En un mismo país, en años muy próximos y con instituciones semejantes, se aprobaron reformas energéticas de dimensiones muy diferentes. Al comparar ambos procesos de decisión se desprenden conclusiones sobre cuáles son las variables independientes que explican el carácter incremental de la reforma de 2008 y el radical de la de 2013, así como, en términos más generales, qué explica la profundidad de una reforma estructural y, dicho de manera aún más abstracta, el cambio en cualquier política pública.

Las instituciones que norman la relación Ejecutivo-Legislativo en lo que se refiere a presentación, negociación y aprobación de leyes y reformas prácticamente no variaron entre 2008 y 2013. La única modificación consistió en la facultad del presidente para presentar iniciativas preferentes, aprobada en 2012, y que, de hecho, no fue utilizada en la reforma energética de 2013. Tampoco variaron las reglas fundamentales para integrar el poder Legislativo, por lo que quedó intocado el modelo mixto de legisladores de mayoría relativa y de representación proporcional, que dificulta que cualquier partido tenga siquiera mayoría absoluta, ya no digamos calificada.

Así, en ambos casos el partido del presidente en turno no tenía mayoría absoluta en ninguna de las dos cámaras. De hecho, su representación era más o menos semejante, ligeramente por encima del $40 \%$ en cada cámara. En este sentido, la ventaja relativa del PRI en 2013 sobre el PAN en 2008 es que contaba con partidos aliados prácticamente permanentes -PVEM y PANAL- que le permitían alcanzar la mayoría absoluta en la Cámara de Diputados, pero no en la de Senadores. Esta ventaja, empero, pierde importancia si se considera que la iniciativa presidencial de 2013 consistía en 
reformas constitucionales, cuya aprobación requería dos terceras partes de ambas cámaras y la mitad más uno de los congresos estatales, lo que obligaba al PRI a construir una mayoría mucho más amplia que la que tenía con PVEM y PANAL. Dicho en breve, ni el PAN en 2008 ni el PRI en 2013 tenían los votos siquiera para aprobar reformas a leyes secundarias en ambas cámaras, aun contando a los partidos aliados del PRI en 2013.

En 2008, el presidente Calderón decidió presentar al Congreso una propuesta de reforma incremental, que solamente incluía modificaciones a leyes secundarias; en 2013, el presidente Peña optó por una iniciativa de reforma radical, que incluía cambios a la Constitución. Calderón presentó su iniciativa tras los fracasos de sus antecesores Zedillo y Fox para concretar reformas energéticas; Peña presentó su iniciativa tras los mismos fracasos, pero además conociendo las dificultades que enfrentó Calderón en 2008 para sacar adelante una reforma incremental. Así, en contextos adversos, Calderón propuso modificaciones incrementales y Peña Nieto radicales.

Mientras la iniciativa presidencial que culminó en la reforma de 2008 fue presentada de manera unilateral por el presidente, la de 2013 fue impulsada en un contexto atravesado por la colaboración entre los tres principales partidos políticos, expresado en el Pacto por México, aunque no se presentó estrictamente dentro de este acuerdo. La decisión legislativa de aprobar la reforma energética de 2013 se explica en buena medida porque, incluso antes de presentar la iniciativa, ésta contaba ya con el respaldo de distintos actores programáticos dentro del Legislativo, es decir, "actores colectivos que comparten ideas de políticas y compiten por la autoridad legítima sobre la formulación de políticas sectoriales" ${ }^{48}$ que pertenecían a partidos distintos, lo que prácticamente garantizaba su aprobación.

Al final del día, tanto el presidente y el PAN en 2008 como el presidente y el PRI en 2013 sumaron públicamente a su principal partido opositor a la reforma, el PRI y el PAN, respectivamente. La diferencia sustantiva fue que los primeros lo hicieron una vez

${ }^{48}$ Hassenteufel, Smyrl, Genieys y Moreno-Fuentes, op. cit., p. 518. 
presentada la iniciativa y los segundos antes de que se presentara. Esto trajo consecuencias de gran trascendencia, pues mientras Calderón presentó una iniciativa de reforma incremental con serias dudas sobre si sería aprobada o no, Peña Nieto presentó una propuesta de reforma radical que sabía, de antemano, que tenía enormes probabilidades de salir adelante.

Cuando el PRI fue oposición, restó fuerza a los cambios que propuso Calderón en materia energética; en cambio, cuando el PAN fue oposición, profundizó las modificaciones propuestas por el presidente. Tal diferencia en la actitud de la oposición se relaciona con las estrategias legislativas del PRI en 2008 y el PAN en 2013: mucho menos presta a la colaboración con el Ejecutivo la primera que la segunda. De hecho, la estrategia del PRI de aminorar el calado de las iniciativas de Calderón no sólo se visibilizó con la reforma energética, sino también con otras durante sexenio, como la fiscal y la política. A su vez, en 2013, el PAN mostró disposición para colaborar con el Ejecutivo en la reforma energética, y además en prácticamente todas las reformas presentadas por Peña Nieto aquel año. ${ }^{49}$

\section{Conclusiones}

La comparación entre los procesos de decisión legislativa de la reforma energética incremental de 2008 y de la radical de 2013 aporta elementos para sostener que los agentes explican de manera mucho más robusta que las instituciones el carácter incremental o radical de una reforma estructural y, en términos más abstractos, del cambio en cualquier política pública.

La mayor parte de los planteamientos neoinstitucionalistas tienen dificultades para explicar el cambio en general y más dificultades aún para explicar los detalles del cambio, como el carácter incremental o radical de éste. Como se ha expuesto, entre 2008 y 2013 prácticamente no hubo modificaciones en las instituciones

${ }^{49}$ Como se ha dicho, no apoyó la reforma fiscal, pero ésta no es una reforma constitucional. 
que norman la relación Ejecutivo-Legislativo ni las relativas a la integración de ambas cámaras. De March y $\mathrm{Olsen}^{50}$ se desprende que hay un número limitado de opciones de políticas, definido por las instituciones. En los casos bajo estudio, con instituciones casi idénticas, se decidieron reformas energéticas radicalmente distintas en cuanto a profundidad, lo que implica que las instituciones no definieron los límites de las opciones de políticas.

Según Heclo, ${ }^{51}$ el tipo de red define el tipo de política, lo que dificulta explicar cambios radicales en una política pública cuando hay continuidad estructural e institucional, tal como ocurre en este caso. Mahoney y Thelen ${ }^{52}$ destacan cómo las instituciones generan contextos propicios para el cambio. En los casos bajo estudio, el carácter incremental de la reforma energética de 2008 y el radical de la de 2013 no puede explicarse desde esta última perspectiva teórica, pues las instituciones no cambiaron y por ende tampoco lo hicieron los contextos propicios para el cambio, lo que no obstó para que éste ocurriera.

Como sostienen Bachrach y Baratz, ${ }^{53}$ la forma como se define un asunto limita las opciones de acción disponibles y, por ende, condiciona las decisiones futuras, lo que beneficia a algunos y perjudica a otros. La definición del asunto la hacen los agentes y es en buena medida esto lo que explica que se haya concretado una reforma incremental en 2008 y una radical en 2013. Ni el PAN en 2008 ni el PRI en 2013 tenían los votos suficientes para aprobar, en solitario, ni siquiera leyes secundarias o reformas a éstas, para no hablar de modificaciones constitucionales. En 2013 se concretó una reforma energética radical porque el presidente y el PRI definieron la reforma energética como un proyecto común con el PAN, partido al que sumaron a una lógica de colaboración permanente con el Ejecutivo, además de sus aliados tradicionales, PVEM y PANAL. El PAN, por su parte, no se limitó a apoyar la iniciativa presidencial, sino que impulsó su profundización. Esto le permitió

\footnotetext{
${ }^{50}$ March y Olsen, op. cit.

51 Heclo, op. cit.

52 Mahoney y Thelen, op. cit.

53 Bachrach y Baratz, op. cit.
} 
al Ejecutivo presentar al Congreso una reforma radical con altas probabilidades de ser aprobada, porque antes de hacerlo construyó, dentro del Legislativo, una coalición suficientemente amplia de "actores programáticos" dispuestos a aprobarla. ${ }^{54}$ En 2008 fue posible una reforma incremental en alguna medida porque el presidente y el PAN definieron la reforma energética como una asunto exclusivo de ellos, incorporando al PRI hasta una vez presentada la iniciativa. En aquella ocasión, el PRI se sumó a las discusiones para tratar de restarle profundidad a la iniciativa presidencial. Calderón había presentado una propuesta de reforma incremental pues sabía que no tenía los votos necesarios para sacar adelante una constitucional, toda vez que no contaba con un respaldo suficientemente amplio de actores dentro del Congreso.

Los autores que explican el contenido de una política pública sobre todo en función de los agentes no soslayan la importancia de las instituciones. La comparación entre los dos casos en cuestión muestra que las instituciones sí pesan, pero como marco de la reforma estructural bajo estudio, no como causa. Tanto la definición del asunto -con las posibilidades que abrió y cerró- como la construcción de la alianza de actores programáticos que impulsaron la reforma, se dieron en un determinado contexto institucional, pero el mismo no explica el resultado tan distinto en 2008 y en 2013, por el sencillo hecho de que la profundidad de ambas reformas presenta una enorme varianza, mientras que el contexto institucional permaneció casi idéntico.

De entre quienes sostienen que los agentes explican -mucho más que las instituciones- el carácter incremental o radical de una política pública, el autor cuyo planteamiento queda más apuntalado a partir del ejercicio comparado en cuestión es Schattschneider. ${ }^{55}$ La variable más robusta para explicar una reforma incremental en 2008 y una radical en 2013 es la estrategia de los agentes. En 2008, la estrategia del presidente Calderón y el PAN cumplió con su objetivo de aprobar una reforma incremental, pero ni siquiera intentó

\footnotetext{
${ }^{54}$ Siguiendo a Hassenteufel, Smyrl, Genieys y Moreno-Fuentes, op. cit.

${ }^{55}$ Schattschneider, op. cit.
} 
ampliar los alcances de lo posible con nuevas estrategias que le permitieran conseguir los apoyos necesarios para una reforma radical. A su vez, la estrategia del PRI en 2008 fue complicar la aprobación incluso de una reforma incremental, lo que sugiere que su papel como oposición habría sido aún más duro si se hubiera presentado una reforma radical. En 2013, en contraste, la estrategia del presidente Peña Nieto y el PRI consistió en ampliar los límites de lo posible mediante el Pacto por México, acuerdo que no había propuesto ninguno de los otros presidentes que enfrentaron gobiernos divididos. La puesta en marcha de este Pacto, que contaba con la participación del PAN, explica en buena medida el ánimo de colaboración legislativa entre PRI y PAN, que finalmente derivó en la reforma energética de 2013.

\section{BibLIOGRAFÍA}

Bachrach, P. y M. Baratz, "Two Faces of Power”, The American Political Science Review, vol. 56, núm. 4, 1962, pp. 947-952.

Cámara de Diputados, "Aprueban diputados, en lo general, minuta de reforma energética y continúan discusión de reservas a todos los artículos, que presentaron 102 legisladores de PRD, MC y PT", 2013. Consultado el 22 de abril de 2014, en http://www3.diputados.gob.mx/ camara/005_comunicacion/b_agencia_de_noticias/009_2013/12_ diciembre/11_11/5309_aprueban_diputados_en_lo_general_minuta_de_reforma_energetica_y_continuan_discusion_de_reservas_a_ todos_los_articulos_que_presentaron_102_legisladores_de_prd_ mc_y_pt

Casar, M., "Las bases político-institucionales del poder presidencial en México”, Política y Gobierno, vol. 3, núm. 1, 1996, pp. 61-92.

—_ "Los gobiernos sin mayoría en México: 1997-2006”, Política y Gobierno, vol. 15, núm. 2, 2008, pp. 221-270.

e I. Marván (coords.), Reformar sin mayorias, México, Taurus, 2014.

Dahl, R., "The Concept of Power", Behavioral Science, vol. 2, núm. 3, 1957, pp. 201-215.

Diario Oficial de la Federación, 28 de noviembre de 2008. Consultado el 7 de 
septiembre de 2015, en http:/ / dof.gob.mx/nota_to_doc.php?codnota $=5070925$

, 20 de diciembre de 2013. Consultado el 7 de septiembre de 2015, en http://www.dof.gob.mx/nota_detalle.php?codigo=532746 $3 \&$ fecha $=20 / 12 / 2013$

, 11 de agosto de 2014a. Consultado el 7 de septiembre de 2015, en http:/ / www.dof.gob.mx/nota_to_doc.php?codnota=5355985

—, 11 de agosto de 2014b. Consultado el 7 de septiembre de 2015, en http:/ / www.dof.gob.mx/nota_to_doc.php?codnota=5355988

— 11 de agosto de 2014c. Consultado el 7 de septiembre de 2015, en http:/ / www.dof.gob.mx/nota_to_doc.php?codnota=5355982

El Universal, "Reforma energética propuesta por EPN", 2013a. Consultado el 10 de septiembre de 2015, en http:/ / www.redpolitica.mx/contenido/documento-integro-reforma-energetica-propuesta-por-epn

-, "Propuesta de reforma energética del PRD", 2013b. Consultado el 10 de septiembre de 2015, en http:/ / www.redpolitica.mx/contenido/documento-propuesta-de-reforma-energetica-del-prd

Giddens, A., La constitución de la sociedad, Buenos Aires, Amorrortu, 2011.

Gil, G., "La reforma petrolera de 2008 en México. Aspectos jurídicos, efectos económicos", Este País, núm. 214, 2009, pp. 39-43.

Grupo parlamentario del PAN en el Senado de la República, "Iniciativa de reforma a los artículos 25, 27 y 28 de la Constitución Política de los Estados Unidos Mexicanos", 2013. Consultado el 10 de septiembre de 2015, en http:/ /www.pan.senado.gob.mx/wp-content/uploads/2013/ 07/Inic_PAN_art.25-27-y-28-Const.pdf

Hall, P. y R. Taylor, "Political Science and the Three New Institutionalisms”, Political Studies, núm. 44, 1996, pp. 936-957.

Hassenteufel, P., M., Smyrl, W. Genieys y F. Moreno-Fuentes, "Programmatic Actors and the Transformation of European Health Care States", Journal of Health Politics, Policy and Law, vol. 35, núm. 4, 2010, pp. 517-538.

Heclo, H., "Las redes de asuntos y el poder Ejecutivo”, en L. Aguilar (ed.), Problemas públicos y agenda de gobierno, México, Miguel Ángel Porrúa, 2000, pp. 257-284.

Lindblom, C., "La ciencia de 'salir del paso", en L. Aguilar (ed.), La hechura de las políticas, México, Miguel Ángel Porrúa, 2000, pp. 201-225. 
— "Todavía tratando de salir del paso", en L. Aguilar (ed.), La hechura de las políticas, México, Miguel Ángel Porrúa, 2000, pp. 227-254. Magar, E. y V. Romero, "México: reformas pese a un gobierno dividido", Revista de Ciencia Política, vol. 28, núm. 1, 2008, pp. 265-285.

Mahoney, J. y K. Thelen, “A Theory of Gradual Institutional Change”, en J. Mahoney y K. Thelen (eds.), Explaining Institutional Change, Nueva York, Cambridge University Press, 2010, pp. 1-37.

March, J. y J. Olsen, Rediscovering Institutions: The Organizational Basis of Politics, Nueva York, The Free Press, 1989.

Nacif, B., The Fall of the Dominant Presidency, México, CIDE, 2006.

North, D., Instituciones, cambio institucional y desempeño económico, México, Fondo de Cultura Económica, 1993.

- "Toward a Theory of Institucional Change", en W., Barnett, N., Schofield y M. Hinich, (eds.), Political Economy: Institutions, Competition, and Representation, Cambridge, Cambridge University Press, 1993, pp. 61-69.

Przeworski, A. y H. Teune, The Logic of Comparative Social Inquiry, Nueva York, Wiley, 1970.

Quade, E., Analysis for Public Decisions, Nueva York, American Elsevier, 1975.

Sabatier, P. y H. Jenkins-Smith, "The Advocacy Coalition Framework", en P. Sabatier (ed.), Theories of the Policy Process, Boulder, Westview Press, 1999, pp. 117-166.

Schattschneider, E., El pueblo semisoberano, México, Unión Tipográfica Hispanoamericana, 1967.

Senado de la República, "Aprueba Senado en lo general reforma energética”, 2013. Consultado el 22 de abril de 2014, en http: / / comunicacion. senado.gob.mx/index.php/periodo-ordinario/boletines/10311-bo letin-861-aprueba-senado-en-lo-general-reforma-energetica.html 